\title{
Affective Film Dataset from India (AFDI): Creation and Validation with an Indian
}

\author{
Sample
}

\author{
Sudhakar Mishra ${ }^{1 *}$, Narayanan Srinivasan ${ }^{2,3^{*}}$, Mohammad \\ Asif $^{1}$ and Uma Shanker Tiwary ${ }^{1}$ \\ $1^{*}$ Center for Cognitive Computing, Indian Institute of \\ Information Technology Allahabad, Jhalwa, Prayagraj, 211012, \\ Uttar Pradesh, India. \\ ${ }^{2}$ Department of Cognitive Science, Indian Institute of Technology \\ Kanpur, Kanpur, 208016, Uttar Pradesh, India. \\ ${ }^{3}$ Centre of Behavioural and Cognitive Sciences, University of \\ Allahabad, Street, Prayagraj, 211002, Uttar Pradesh, India.
}

*Corresponding author(s). E-mail(s): rs163@iiita.ac.in; nsrini@iitk.ac.in;

Contributing authors: pse2017001@iiita.ac.in; ust@iiita.ac.in;

\begin{abstract}
We describe the creation of an affective film dataset for researchers interested in studying a spectrum of emotional experiences. We followed a two stage process. In the first stage, two hundred twenty-two video clips with 60 -seconds long duration were rated in the lab by 407 participants. Based on the selection criteria, 69 audio-visual clips were selected for the second stage. These selected affective clips are then presented to 271 participants. Participants rated these clips on rating scales and categorized them into emotion categories. We clustered these emotional ratings into six categories, calculated the probability of eliciting emotion categories for each stimulus and compared the probability results with datasets created with the Western population. Given that part of the dataset consisting of videos from the west have been rated earlier, we compared ratings by Indian and Western participants on that subset. Even with some differences in methodology and analyses, we did
\end{abstract}


not observe significant differences in ratings of different scales. Interestingly, the ratings to Indian stimuli by Indian participants tended to be significantly higher in arousal, dominance and familiarity than the ratings to western stimuli by Indian participants. The dataset we have created comprises film clips based on Indian and western content, making it flexible for use in Indian or western cultures. We hope that this dataset emotion researchers with a more diverse set of emotional movie clips accompanied by emotional ratings and other quantitative measures.

\section{Introduction}

Emotion research has relied predominantly on static pictures of facial expressions and scenes. Given that our minds and brains function 'in the wild', where stimuli are dynamic, multimodal, crowded, and context-dependent, the usage of static stimuli is a concern. Affective films that are more ecologically valid than affective pictures and can approximate real-life scenarios emerged over two decades ago (Gross \& Levenson, 1995; Philippot, 1993). The meaning of stimuli in the real world is typically dependent on their dynamic context, which accrues over minutes to hours. Films have a higher degree of ecological validity as emotions are often evoked by dynamic visual and auditory stimuli external to the individual. Film snippets provide a multimodal experience for the viewer, engaging both the visual and audio sensory systems simultaneously and coherently. They can induce fundamental emotions like fear or disgust as well as more complicated or subtle emotions (Ray \& Gross, 2007; Schaefer, Nils, Sanchez, \& Philippot, 2010).

In the 1990s, researchers systematically explored the efficacy of movie clips for emotional induction and constructed validated datasets (Gross \& Levenson, 1995; Philippot, 1993). In recent years, significant attempts have been made to develop larger and more comprehensive datasets of short movies (GabertQuillen, Bartolini, Abravanel, \& Sanislow, 2015; Gilman et al., 2017; Jenkins \& Andrewes, 2012; Ray \& Gross, 2007; Samson, Kreibig, Soderstrom, Wade, \& Gross, 2016; Schaefer et al., 2010; Von Leupoldt et al., 2007). Each dataset has its own set of strengths and has attempted to fill the gaps in the availability of standardized dynamic affective stimuli sets. Nonetheless, many past and recent studies are still based on a relatively smaller number of clips that may limit generalizability. In addition, many targeted a smaller number of emotional states that may not allow us to study a wide range of emotions.

One factor of importance, especially in studying the role of contextual information, is the duration of the emotional video itself. A survey of available affective film clip libraries reveals a wide range of stimulus durations, ranging from under a minute (Carvalho, Leite, Galdo-Álvarez, \& Gonçalves, 2012; Samson et al., 2016) to over seven minutes (Schaefer et al., 2010). Moreover, most databases (Gross \& Levenson, 1995; Hewig et al., 2005; Schaefer et al., 2010) have significant variability in the time duration of affective film clips within a 
given database. Since there is no clear criterion for the duration of stimulus necessary to elicit an emotion, most authors of previous databases utilized the integrity of the scene as a criterion or expert's opinion for suitable durations, resulting in a significant variation in film durations within and across numerous databases (Baveye, Dellandrea, Chamaret, \& Chen, 2015; Gabert-Quillen et al., 2015; Gilman et al., 2017; Gross \& Levenson, 1995; Hewig et al., 2005; Jenkins \& Andrewes, 2012). This duration factor also depends on the type of targeted emotions. For instance, stimuli with a shorter length are generally inadequate in eliciting complex emotions. On the flip side, the longer-duration stimuli have the disadvantage of heterogeneity of multiple emotions and temporal localization of emotion in a given time interval. Hence, a film dataset in which the film clips have a constant duration would be ideal for eliciting simple and complex emotions.

Another problem with earlier film datasets is the film categories that need to be included and the affective states that need to be targeted. Generally, datasets include thrillers and horror films to induce fear and disgust, covering the negative affect range. There is less unanimity in the selection of film categories for eliciting good feelings. The conventional approach has been to include clips from film comedies and/or stand-up humour to target positive affective states such as joy, happiness, and amusement, which are the most commonly reported emotions (Gilman et al., 2017).

We reduced experimenter bias in the initial screening of emotional stimuli by relying on computer algorithms to search multimedia videos for affective terms, followed by automatic content analysis to calculate the valence and arousal from audio-visual features, which are subsequently utilized to select the candidate film clips (Mishra, Srinivasan, \& Tiwary, 2022). Rather than restricting to a small number of emotional categories, we asked the participants about the emotion category they felt during viewing clips to reduce experimenter bias further.

Moreover, emotion studies on non-native English-speaking populations in the Indian sub-continent have mostly relied on scenes (IAPS) and images of facial expressions (Lohani, Gupta, \& Srinivasan, 2013; Srinivasan \& Gupta, 2010; Srinivasan \& Kumar, 2012). One study (Dhaka \& Kashyap, 2017) used film clips to compare emotion elicitation with pictures. However, this study used film clips from an already available stimuli repository containing clips from the West. It is reported in several studies that eastern cultures differ in their feelings and expressions of emotions from western cultures (Lim, 2016). Moreover, this difference is also reflected in the songs and movie making, which have targeted audience (Hogan, 2009). Additionally, a significant number of studies have been done on the Western, educated, industrialized, rich and democratic (WEIRD) population, which poses questions regarding the generalizability of results from these studies to other cultures and societies (Henrich, Heine, \& Norenzayan, 2010). Given that India is a large country that shares $17.5 \%$ of the world population, there is a need to create a dataset of film clips that is suitable for the population of the Indian subcontinent but also could 
be used elsewhere. Hence, we created a dataset containing some clips from India, but we did not restrict ourselves to only Indian film clips so that English speakers elsewhere could also use parts of the same dataset in their research.

\section{Method}

This study was not preregistered.

\section{Stimuli Collection, Selection and Validation}

\subsection{Stimuli Collection}

The procedure for stimuli collection is explained elsewhere (Mishra et al., 2022). Briefly, we crawled YouTube to collect candidate emotional stimuli. Multimedia content analysis including analysis of user comments, analysis of audio and visual cinematic features on these stimuli was performed, which were eventually used to generate an initial set of two hundred and twenty two videos.

\subsection{Stimulus Validation}

\subsubsection{Participants}

All the participants were recruited from the Indian Institute of Information Technology, Allahabad, India. Students from the institution were recruited through advertisements and presentations in some classes. Participants were provided laboratory course credit for participation, and they were also provided with an e-certificate signed by PI as an appreciation for taking part in a research study. Participants self reported that they do not have any history of mental disorders. The study was approved by the institutional review board, University of Allahabad (protocol code 2017-100 approved on Dec 8, 2017). As part of the approval protocol, all stimuli were submitted to the review board and were approved for presentation in the study. Although English is not the native language for the participants, the language of instruction in their schooling was English. They also commonly watch English movies and listen to western music.

In total, six hundred seventy-eight volunteers participated in the study (details about participants are shown in the table-1). The gender ratio of participants was not balanced with more male participants due to the much higher number of male students (predominantly engineering undergraduates) in the institution. The validation study had 574 male and 104 female participants. Only a small number of participants were left-handed (36 out of 678). Participants spoke a wide variety of native languages (29 languages), with Hindi being the most dominant native language given the institution's location in the Northern part of India (as shown in the table-2). Hindi speakers $(\mathrm{N}=537)$ were followed by Telugu speakers from the Southern part of India $(\mathrm{N}=78)$, 
and the sample also included a small number of Punjabi, Marathi, and Bengali speakers. All of them also could converse in Hindi to a more or less extent.

\begin{tabular}{c|clcccc} 
Stage & Partic ipants & $\begin{array}{l}\text { Age } \\
\text { mean(sd) }\end{array}$ & Male & Female & Age_M & Age_F \\
\hline First & 407 & $20.88(2.27)$ & 343 & 64 & $20.86(2.15)$ & $20.98(2.86)$ \\
Second & 271 & $20.37(1.57)$ & 231 & 40 & $20.28(1.64)$ & $20.84(1)$ \\
\hline
\end{tabular}

Table 1: Demographics of participants

\begin{tabular}{c|cccccc} 
Stage & HINDI & PUNJABI & TELUGU & MALAYALAM & BENGALI & Others \\
\hline First & 315 & 14 & 43 & 6 & 6 & 23 \\
Second & 222 & 7 & 35 & 0 & 3 & 4 \\
\hline
\end{tabular}

Table 2: Statistics of the native language of participants

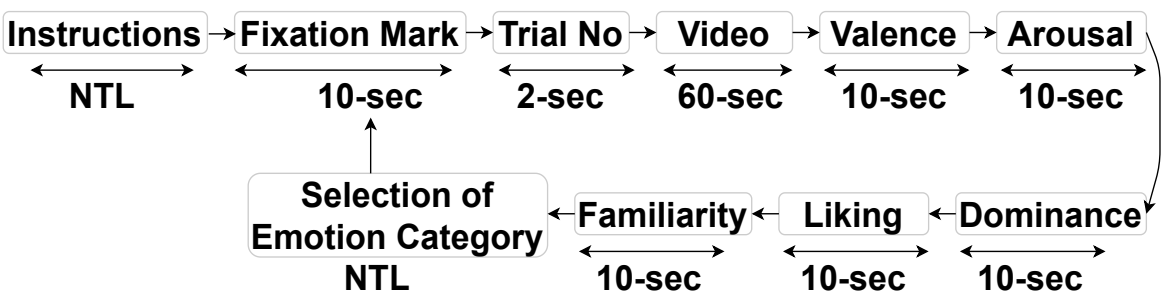

Fig. 1: Experiment Paradigm: NTL: No time limit

\subsubsection{Experiment Paradigm}

Before the experiment, participants were given a presentation on a projector screen and briefed about the experiment. They were also told how to perform the experiment (i.e. how the experiment will progress and how should they give their ratings and responses). Scales were also explained to participants, and after no query from the student's side, they were randomly assigned a computer for the experiment. The right side block diagram of the Figure S1 depicts the lab configuration used to carry out stimuli validation. The distance between participants was around 2 meters giving enough space between participants and lights were off.

The whole rating study was divided into two stages, given the complexity of validating a large number of videos with an adequate number of raters. For the initial set of 222 video stimuli, we made 14 blocks with 16 videos in each block. In the second stage, we had 69 video stimuli, which were divided into eight blocks. The blocks were recreated for every batch of participants by reassigning videos randomly and automatically to minimize potential grouping or 
selection biases. Due to the limited allotted time duration for the experiment, we did not include emotion category classification in the first stage of rating validation.

Participants were given a training session with three stimuli so that they get familiar with the experimental procedure. Their doubts, if any were clarified after training and then they were allowed to start the experiment. The experiment followed the procedure depicted in the figure-1. During the first and second stages, each participant saw 16 and 9 video stimuli respectively followed by rating scales to record the self-assessment ratings. Participants were given a list of emotion categories during the second stage only (figure-1). This list of emotions was generated based on selecting ratings on valence and arousal scales (see supplementary material for scale rating instructions). Participants were also given the choice to propose their own emotion category if they did not find a suitable emotion in the list (during the second stage). After the eighth and fifth videos (first and second stages, respectively), participants were given a choice to relax, and the experiment paused until they pressed any key to resume the experiment.

We used five self-assessment rating scales to get the ratings of subjective experience. The valence scale ranged from unpleasant (1) to pleasant (9). To help participants rate the valence scale, SAM pictures depicting unpleasant to pleasant faces were shown above the rating scale. The arousal scale ranged from inactive (1) to active (9). Again, SAM pictures depicting inactive or low arousal to active or high arousal were shown above the rating scale. The dominance scale ranged from submissive (1) to dominant (9). The SAM picture depicted the range of dominance with the size of the cartoon in the picture. How much participants liked a stimulus was rated on the liking scale ranging from least (1) to fair (3) to much (5). The familiarity of the content was rated on the familiarity scale ranging from least (1) to fair (3) to much (5). Unlike first stage, in the second stage participants were also asked to label the stimulus with the emotion category that suitably described their emotional feelings. The list of emotions presented to the participants are included in the supplementary material. The Figure S2 depicts how the scales were presented. Instructions for rating the scales are also provided in supplementary material.

\subsubsection{Stimuli Selection}

After obtaining the scale ratings from 407 participants in the first stage of the validation study (from (Mishra et al., 2022)), we performed two operations. First, the emotion labels were tentatively assigned to stimuli to aid the stimuli selection for the second stage. The list of emotion words and their corresponding ratings reported in an earlier study (Warriner, Kuperman, \& Brysbaert, 2013) was considered for the emotional labelling. The Euclidean distance between mean valence-arousal ratings by our participants and mean valence-arousal ratings of seventy-two emotion words (rated by Warriner et al. (2013)) was calculated (the same list of seventy-two emotion words is used for the initial selection of 222 stimuli in our earlier study). Please note that 
this assignment was tentative and only helped us to select stimuli with the intention of covering a wide range of emotions. Hence, we followed a simple assumption that if the mean valence and arousal ratings of two film stimuli (in our data) are close (close based on the lowest Euclidean distance) to the same emotion word (in the Warriner list of emotions), these stimuli will more likely elicit similar emotional experience. Following this procedure, each stimulus in the first stage was labelled with an emotion category.

Second, we used participants' self-assessment ratings on valence and arousal scales to screen the video stimuli for the second stage. We calculated average valence and arousal ratings for each stimulus and projected them on the valence-arousal space. The valence and arousal dimensions ranged from 1 to 9 and formed the vector. The magnitude of each vector (representing a stimulus on V-A space) was calculated. This magnitude represents how far the average stimulus ratings are from the center (larger the magnitude implies the stimulus is near the periphery of the $\mathrm{V}$-A space). In addition to the magnitude of the vector, we also considered the standard deviation in participants' ratings for each stimulus. To sum up, the idea behind considering the vector's magnitude is how close the stimuli ratings are towards the perimeter of the V-A space (following the circumplex structure of affect, (Russell, 1980)), and standard deviation tells how consistently participants rated these stimuli. The vector's magnitude and standard deviation were used as features for the k-means clustering algorithm.

We clustered the video stimuli using K-means clustering. We observed that the cluster with a higher magnitude of the vector had less joint valence-arousal standard deviation. The low SD signifies the consistency of valence-arousal ratings for a stimulus. We considered the cluster with higher vector's magnitudes and low joint standard deviations (cluster-1). However, we did not include all of the stimuli from this cluster because they over-represented some emotion categories. Thirty-eight clips were selected from this cluster. Thirty-one film clips were added from another cluster (cluster-2) to include more emotion categories and increase the number of clips for underrepresented emotion categories. However, during the selection of thirty-one stimuli from cluster-2, we still selected stimuli with high magnitude and low standard deviation in cluster-2. We performed a significance test to assess if the set of final sixtynine stimuli had more magnitude and low variance than a randomly selected sixty-nine stimuli (the randomly selected stimuli exclude the final 69 stimuli). The results show that our set of sixty-nine stimuli indeed had higher vector magnitude and low variations than randomly selected stimuli (see the Table S1).

In total, sixty-nine stimuli were selected from the first stage. All the emotion categories from the first stage were considered to create a list of emotions for the second stage of ratings to 69 stimuli. We did not include four words brutality, curiosity, disturbing, and funny. Since participants felt brutality was a violent word and were reluctant to potentially use it (in the pilot), we instead used hate. Instead of funny, happy (a more popular emotion word) 
was included. Other emotion categories from the periphery of the circumplex model (Paltoglou \& Thelwall, 2012) were included. These emotion words were near the emotion words that we got from the first stage. In total, a list of 48 emotion words was included in the second stage of the study (see Table S2 ). The set size can be favourably compared to the number of emotion categories used in earlier studies (see Table S3).

\section{Data Analyses}

\subsection{Data Cleaning}

During the data cleaning, we removed only those participants who a) gave the same response to all the stimuli, for example, 5, 5, 5; b) had not completed the experiment; and c) did not provide some ratings on the rating scales. The percentage of participants excluded from the first and second stages were 1.9\% and $0.3 \%$.

\subsection{Comparison between Indian and Western Ratings on stimuli taken from DEAP dataset}

We compared the methodologies adapted by DEAP dataset developer (Koelstra et al., 2011) and by us (see supplementary material for comparison of methodologies). In the first stage, we had 222 one-minute excerpts, of which 98 were from the DEAP database. In our final set of 69 videos, there were 18 from the DEAP database. This allowed us to compare ratings for those videos from DEAP that are part of our database. We performed two-sample t-tests (if the data satisfied the normality condition) or Wilcoxon rank-sum tests (if the data did not satisfy the normality condition). The Bonferroni correction for multiple hypothesis comparison was used. We also performed Bayesian two-sample independent t-tests to assess how much one hypothesis explained the data in relation to the other. In this case, the null hypothesis was that there was no difference between ratings by Indian and Western participants. We assumed a Cauchy prior distribution with $r=\frac{1}{\sqrt{2}}$.

\subsection{Comparing ratings by Indian participants on Indian and Western stimuli}

One issue that is of interest is potential differences in the way Indian participants rate Indian and western videos. Hence, we performed a Welch two-sample t-test to evaluate the participants' self-assessment ratings on different scales to Western and Indian stimuli. In addition, we also performed a Bayes factor analysis. The null hypothesis states that there is no difference in self-assessment ratings on different scales between the Western and Indian stimuli and, therefore $H_{0}: \delta=0$. The two-sided alternative hypothesis states that the positive and negative values of $\delta$ are possible. $\delta$ was assigned a Cauchy prior distribution with $r=\frac{1}{\sqrt{2}}$ (for all self-assessment scales). 


\subsection{Comparison of emotional categorization}

During the ratings for 69 stimuli, our participants had to select an appropriate emotional category describing their emotional feelings. To compare the probability of emotional categorization from our study with earlier database studies, we had to cluster some emotions to form groups. We grouped emotion categories rated in our dataset into six groups. These groups are tabulated in the table-3. We have grouped disgust with angry emotion. The stimuli that elicited disgust emotion have content related to social and moral violations, which also elicits aggression (Molho, Tybur, Güler, Balliet, \& Hofmann, 2017). Hence, our dataset lacks stimuli for eliciting non-social disgust. We compared our results with the emotion elicitation score reported by Gross and Levenson (1995) and Zupan and Eskritt (2020).

\begin{tabular}{c|l} 
Emotion Groups & Emotion Names \\
\hline Sad & Despondent, Taken Aback, Sad, Ashamed, Dissatisfied, \\
& Melancholic, Miserable, Depressed, Gloomy \\
Astonishment/Surprise & Astonished, Enthusiastic, Adventurous, Excited \\
Afraid/fear & Afraid, Startled, Alarmed, Tense \\
Contentment & Pleased, Contented, Hopeful, Pensive, Calm, Peaceful, Con- \\
& templative, Love, Relaxed \\
Amusement/Happy/Joy & Triumphant, Delighted, Joyous, Happy, Amused, Convinced \\
Anger & Impatient, Angry, Hate, Frustrated, Indignant, Disgust \\
\hline
\end{tabular}

Table 3: Clustering emotions into six clusters which are comparable with six of the eight categories taken by Gross and Levenson (1995) and eleven categories taken by Zupan and Eskritt (2020). Although Gross and Levenson (1995) and Zupan and Eskritt (2020) had more categories, due to lack of some corresponding emotional categories in our dataset, we were not able to create more than six groups.

\section{Results}

\subsection{Representation of ratings for sixty-nine stimuli}

As a result of our selection criteria described in section-2.2.3, we had 69 stimuli. The descriptive statistics on the rating scales are described in Table S4. The figure-2 depicts that these 69 stimuli cover the whole valence and arousal space. However, more stimuli are available in the HVHA and LVLA quadrants. The least number of stimuli are available in the HVLA quadrant.

\subsection{Comparison of ratings of Indian and Western participants for common videos between DEAP dataset and our dataset}

Independent sample t-tests were performed separately for each video and separately for valence, arousal, and dominance to compare ratings from western 


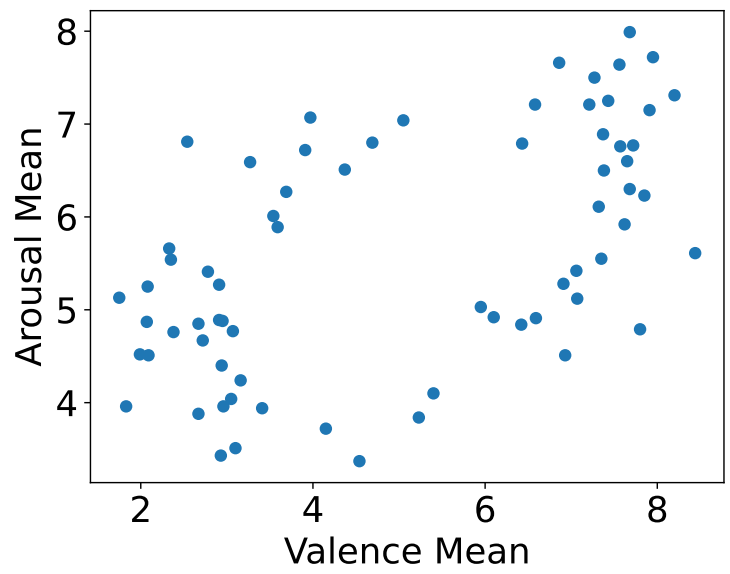

Fig. 2: Representation of 69 stimuli after the second stage. Each dot represents a video stimulus. The mean valence-arousal vector is plotted for 69 video clips, which were selected based on the criteria described in the section-2.2.3.

participants and Indian participants on the common set of stimuli between the DEAP database and our database. We found that for the majority of the stimuli (98 in total), the ratings by western and Indian participants were not significantly different. After correcting for multiple comparisons, we had only one and three videos out of ninety-eight that showed a significant difference in valence and arousal ratings, respectively, between Indian and Western samples. The results indicate that the ratings were not significantly different for almost all the common DEAP videos and give us confidence in the methods employed in the current study.

We considered mean ratings by western and Indian participants of each video and performed Bayesian two-sample independent t-tests. The Bayesian analysis was performed by assuming the Cauchy prior distribution with the width $=\frac{1}{\sqrt{2}}$. The null hypothesis was that there was no difference in ratings between Western and Indian participants. For all the scales, the evidence in favour of the Null hypothesis was moderate, and the BF ranged from 5 to 8. Further, we also considered only those DEAP stimuli which were included in our final set (18). Again, the results showed evidence in favour of the Null hypothesis for all the scales (valence, arousal, and dominance).

In summary, the ratings by Western Participants and Indian Participants on self-assessment scales for the same videos were fairly similar despite the minor methodological differences in the way rating scale data was obtained (for methodological differences, see supplementary material). 


\subsection{Comparison of ratings for Indian and Western video stimuli}

In this analysis, we compared ratings by Indian participants to Indian and Western stimuli. We performed Bayes two-sample independent tests. The twosided alternative hypothesis states that the positive and negative values of $\delta$ are possible. Specifically, $\delta$ was assigned a Cauchy prior distribution with $r=$ $\frac{1}{\sqrt{2}}$ (for all self-assessment scales). We observed no evidence of the alternative hypothesis $\left(H_{1}\right)$ for valence and liking scales. On the other hand, there was moderate evidence for arousal and dominance. For familiarity, the evidence for the alternative hypothesis was strong. Figure-3 shows the prior and posterior distribution of effect size of mean difference (on the left) and robustness check of Bayes factor with different widths of priors (on the right).

Arousal: Top panel (in the figure-3) shows that differences in arousal ratings are approximately 5.8 times more likely to occur under $H_{1}$ than $H_{0}$. The median of the posterior distribution of $\delta$ is -0.598 . However, the spread of credible interval (CI) from -1 to -0.12 induce uncertainty about the effect size. The results are robust to different prior distributions and maintain moderate evidence throughout.

Dominance: Result of the Bayesian 2-sample t-test analysis of the dominance scale (middle panel in the figure-3) shows that $H_{1}$ is 5.13 times more likely to explain the dominance ratings than $H_{0}$. Moreover, this moderate evidence survives the robustness check with different parameters of Cauchy prior distributions. The median of the posterior distribution of $\delta$ is 0.585 . The effect size $(\delta)$ seems uncertain as the credible interval ranges from nearly -1 to -0.11 .

Familiarity: The bottom panel (in the figure-3) shows the Bayesian 2sample t-test analysis of the familiarity scale. Results show that the alternative hypothesis $\left(H_{1}\right)$ is 623 times more likely to explain the data related to familiarity than $H_{0}$. It shows stronger evidence in favour of $H_{1}$, which also persists for different parameter values of the Cauchy prior distribution. The median of the posterior distribution of effect size is nearly -1 , and the credible interval varies from -1.5 to 0.5 .

We also performed frequentist two-sample t-tests. We observed that the valence and liking ratings are not significantly different for Indian and Western videos. As expected, the Indian participants rated familiarity significantly higher for Indian stimuli than the Western stimuli $(t(61.84)=4.63, p<$ $0.0001,95 \% C I=[0.451 .14], d=1.1)$. Ratings of arousal and dominance for Indian stimuli were also significantly higher than the Western stimuli (respectively, $t(56.53)=2.77, \quad p=0.007,95 \% C I=\left[\begin{array}{ll}0.22 & 1.4\end{array}\right], d=0.68$ and $t(44.95)=2.54, p=0.01,95 \% C I=[0.131 .11], d=0.67)$.

\subsection{Probability of eliciting an emotional cluster}

We considered two stimuli with the best probability from the set stimuli for comparison with (Gross \& Levenson, 1995). We observed that categories assigned to stimuli by our participants are comparable with those provided by 

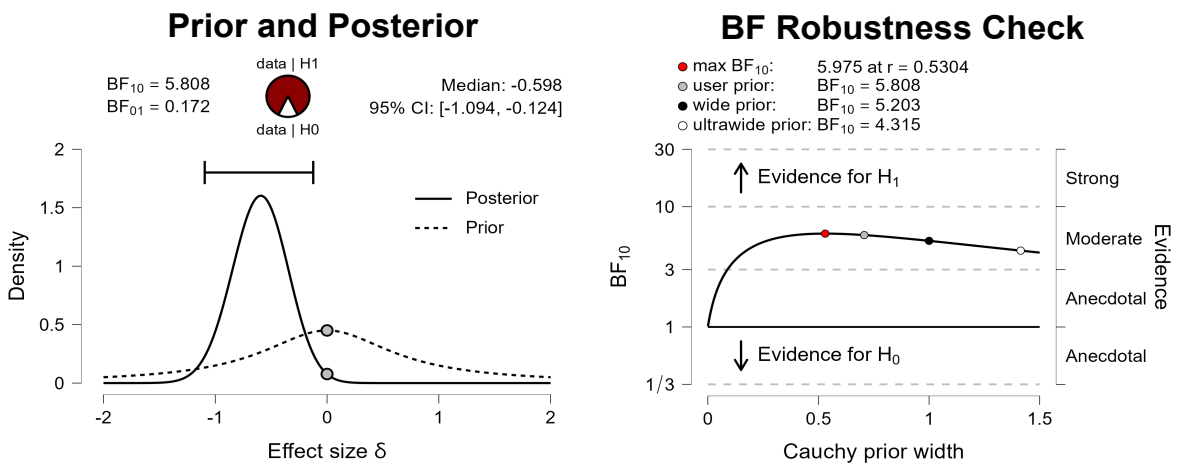

(a)
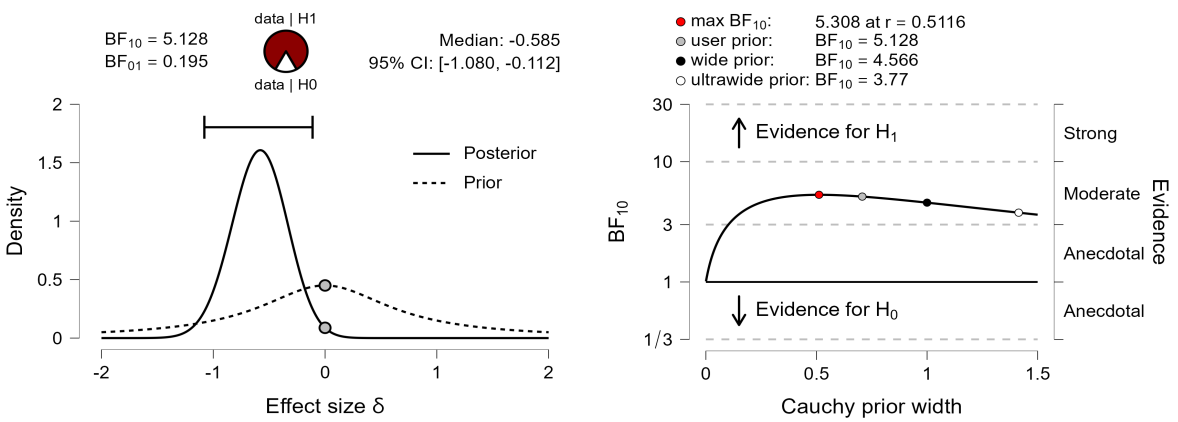

(b)
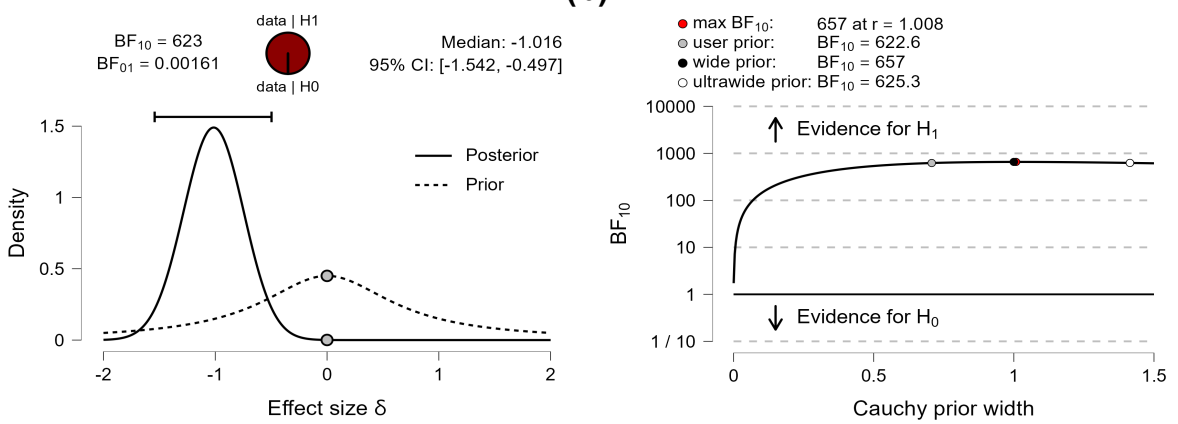

(c)

Fig. 3: Comparing scale ratings between Indian and Western Stimuli: Bayes two-sample Independent analysis was performed. The left figures show prior and posterior distributions of effect size, whereas right figures show the robustness check of Bayes factors (BF) with different parameter settings of prior distributions. Left figures are annotated with the median effect size along with credible interval (CI) and BF (10: evidence of alternative in relation to null hypothesis; 01: evidence of null in relation to alternative hypothesis). The pi-chart is the graphical representation of the BF. A higher coloured slice means stronger evidence for the alternative hypothesis. Annotation in the right side figures shows $\mathrm{BF}$ with different width values of the Cauchy prior distribution. (a) Arousal, (b) dominance, (c) familiarity. 
Gross and Levenson (1995) (see Table S5). For instance, hit rate reported by Gross and Levenson (1995) for the Sadness category was $0.9 \& 0.7$ for the two stimuli. In our analysis, cluster-1 closely resembles the Sadness category, and the probability of reporting Sadness for the two stimuli was 0.7. For the Surprise category the reported hit score by Gross and Levenson (1995) was 0.74 \& 0.67. In comparison, cluster-2 in our analysis resembled the Surprise category with the elicitation probability of $0.68 \& 0.56$. Category-3 in our analysis is related to the Fear category. For Fear category the hit rate reported by Gross and Levenson (1995) was $0.71 \& 0.59$ and the elicitation probability calculated in our analysis for two stimuli were $0.64 \& 0.56$. Contentment category taken by Gross and Levenson (1995) were related with the cluster-4 in our analysis. The probability of eliciting Contentment and Peace related emotions in our analysis was $0.56 \& 0.54$ compared to the reported hit rate by Gross and Levenson (1995) was $0.58 \& 0.43$. For cluster-5, the calculated probability in our analysis was $0.86 \& 0.7$ in comparison to the reported hit rate for Amusement category by Gross and Levenson (1995) was $0.93 \& 0.84$. The hit rate for Angry category was lowest in the ratings by Gross and Levenson (1995) (0.41 \& 0.22) and the cluster- 6 in our study, which is related with the Angry category had the emotion probability $0.48 \& 0.39$ for two stimuli (see table- 4 ). The results indicate that the probability of emotional categorization for the videos in our dataset is similar to those from earlier data sets Gross and Levenson (1995).

We also compared our results with the recent rating study by Zupan and Eskritt (2020). Like, the comparison with ratings by Gross and Levenson (1995), our results are comparable with the recently reported rating results. For instance, for sad emotions, the discreteness score reported by Zupan and Eskritt (2020) and the probability reported in our study was approximately 0.7. Likewise, for the afraid category, the score reported in a study by Zupan and Eskritt (2020) and our study was $\sim 0.6$. For the amusement category, the probability score obtained in our study was 0.86 compared to 0.74 by Zupan and Eskritt (2020). However, for anger category Zupan and Eskritt (2020) reported higher score (0.61) than reported in our study (0.48).

Unlike (Gross \& Levenson, 1995) and (Zupan \& Eskritt, 2020), we report more number of videos which can elicit the reported emotion categories. For instance, for the sad cluster, we have 25 stimuli for which the probability ranges from 0.3 to 0.71 . Five stimuli are enlisted under the astonishment/surprise emotion cluster with the probability ranging from 0.43 to 0.68 . Afraid/fear cluster had eight stimuli with a range of probabilities from 0.37 to 0.64 . The probability ranged from 0.32 to 0.56 for seven stimuli under the contentment emotional category. For amusement/happy/joy emotion cluster, twenty-one stimuli are included with probability ranging from 0.39 to 0.86 . For anger cluster, three stimuli are included with a range of probabilities from 0.34 to 0.48 . 


\section{Discussion}

We started our study with 222 multimedia video stimuli, which were obtained using multimedia content analysis described in (Mishra et al., 2022). Given the increasing amount of multimedia content on the internet, such a screening tool would be useful to make an initial selection of videos and manually handpicking a few videos can be potentially biased. We then selected 69 stimuli from this larger set, which was rated by 271 participants. From the perspective of cultural origin, the set includes 27 film clips from Indian cinema, 41 from Western cinema, and one from Korean Cinema (only visuals, no words).

As shown in the Table S7, most of the earlier database development studies on emotional film stimuli started with an assumed target emotion for a particular film (Gabert-Quillen et al., 2015; Gilman et al., 2017; Gross \& Levenson, 1995; Hewig et al., 2005; Zupan \& Eskritt, 2020). Based on the set of these target emotions, the Likert scales for different emotions were presented to the participants for the ratings. Our method somewhat differs, and we did not assume a pre-specified target emotion for a film clip. Our participants rated their emotional experience on scales for dimensions like valence and arousal, which are regarded as core affect and construct a space in which all the emotional experiences can be represented (although we recognize that the number of dimensions is still debatable). Ratings on the valence and arousal scale have also been obtained and utilized in other studies (Gabert-Quillen et al., 2015; Zupan \& Eskritt, 2020). Finally, instead of restricting participants to a small set of pre-decided target emotions, we gave them an opportunity to label their emotional experiences from a larger list of emotion labels. Participants also had the opportunity to write any emotional category which they did not find in the given emotion list. It should also be noted that all the emotion datasets developed so far do not employ exactly the same method and contain differences as well (see Table S7). Perhaps there is a need for standardization of methods, but we feel that is bound to happen as we explore further and many culturally diverse datasets get created, hopefully from all parts of the world.

To partially validate the database, we also compared our ratings with the ratings obtained for the videos that were in the DEAP database since a significant number of videos is common between the two databases. The results show not only there is no significant difference in ratings but moderate evidence for the null hypothesis (based on Bayesian analysis). This gives us confidence in the methods employed and the ratings from our current study.

Our cultural context influences the way an individual can feel and express an emotional experience (Turner \& Stets, 2005). Emotions are not only biological adaptations but also facilitates and influenced by the interactions in socio-cultural situations. In sociological theories, for example, by Shott (1979), it is argued that an individual first experiences physiological arousal and then use it to label an emotion category. Differences in the level of arousal have been reported in cross-cultural studies (Lim, 2016). For instance, it is observed that the emotional experience in western culture is inclined toward higher arousal, whereas in eastern cultures, relatively low arousal is experienced (Lim, 2016). 
However, in our results, participants felt high arousal for the Indian stimuli compared to western stimuli. In addition, as expected, participants also felt a high familiarity with Indian stimuli than western stimuli. We interpret that due to better familiarity with the cultural context of emotions pertaining to the Indian subcontinent, participants felt higher arousal for Indian stimuli than western stimuli. The influence of culture on dominance and submissiveness in the situation eliciting emotional experience and expression is observed by Butler, Lee, and Gross (2007). At this point, we cannot provide any specific reason why Indian participants on Indian stimuli had felt higher dominance. We speculate that due to living in the same culture, our participants may be able to situate themselves in the situation easily and thus have the feeling of more control.

We also compared the ratings provided by our participants with the previous study done by Gross and Levenson (1995) and Zupan and Eskritt (2020). We observed that the ratings of emotional categories by our participants are comparable with those provided by Gross and Levenson (1995) and Zupan and Eskritt (2020). For instance, Gross and Levenson (1995) reported highest hit rate for the Amusement. Similarly, we have also observed the highest ratings of the emotional cluster related to Amusement. Gross and Levenson (1995) reported that Anger, Contentment and Fear are emotions which are difficult to elicit in the lab environment. Like (Gross \& Levenson, 1995), we also observed lower probability scores for Angry, Contentment and Fear-related stimuli. The discreteness score by a recent study by Zupan and Eskritt (2020) with the probability score by us is also comparable. Overall, after reducing the large list of emotional categories to six clusters for ease of comparison, the results produced by our method are comparable with those provided by methods adapted by Gross and Levenson (1995) and Zupan and Eskritt (2020).

Most of the behavioural and cognitive neuroscience studies are performed among WEIRD populations in the west (Gabert-Quillen et al., 2015; Gilman et al., 2017; Henrich et al., 2010)). This raises questions regarding the generalizability of the results of these studies to other cultures and populations like those in India. So far, we do not have a suitable dataset for use with the Indian population and validated ratings from Indian participants. This makes it difficult to study emotional experience and affective behaviour with dynamic stimuli in India. Our stimuli dataset would be helpful for those who want to do cross-cultural studies since this database is a mixture of videos from at least two broadly different cultures. In an ideal world, we would have a large database with videos from multiple cultures (spanning all the continents), and our hope is that this study will lead toward that endeavour. It should be noted, though, that the Indian samples in our dataset are also restrictive and do not capture all the diversity of a large 1.5 billion population. Hence, future research should diversify the emotional stimuli as well as obtain data from a diverse population of India. Nonetheless, given the presence of videos with non-Indian cinema content in the database, our database could also be used for research in other cultures after suitable validation. 
In summary, this work presents an affective film dataset that can elicit emotions in the lab to better understand the mechanism underlying emotional processing. Although we used a slightly different methodology than described previously to create the video stimuli dataset, our results are comparable with the Western datasets. Since this dataset is based on data collected from an Indian sample, we hope that it can fuel neuro-behavioural research amongst the Indian psychological community to test the generalization of already published results on affect across cultures. 


\begin{tabular}{|c|c|c|}
\hline \multicolumn{3}{|c|}{ Sad } \\
\hline 210 & $\begin{array}{l}\text { The legend of Bhagat Singh (Indian man is being beaten } \\
\text { by English during colonial period) }\end{array}$ & 0.71 \\
\hline 27 & Sarkar (Feeling miserable due to killing his bad brother) & 0.7 \\
\hline \multicolumn{3}{|c|}{ Astonishment (Surprise) } \\
\hline 64 & $\begin{array}{l}\text { Fast \& Furious } 7 \text { (Cars are dropping from the plane } \\
\text { scene) }\end{array}$ & 0.68 \\
\hline 206 & $\begin{array}{l}\text { Iqbal (Actor gets access to play cricket, he was striving } \\
\text { for) }\end{array}$ & 0.56 \\
\hline \multicolumn{3}{|c|}{ Afraid } \\
\hline 10 & $\begin{array}{l}\text { Anaconda (A person all of sudden eaten by Anaconda } \\
\text { in a lake) }\end{array}$ & 0.64 \\
\hline 32 & Titanic (Ship Crash Scene) & 0.56 \\
\hline \multicolumn{3}{|c|}{ Contentment } \\
\hline 53 & Music (Darkest Things) & 0.56 \\
\hline 200 & Calm music with Scenes from Mother Nature & 0.54 \\
\hline \multicolumn{3}{|c|}{ Amusement } \\
\hline 215 & Music (Why This Kolaveri Di) & 0.86 \\
\hline 195 & Chitty Chitty Bang Bang (You Two Scene) & 0.7 \\
\hline \multicolumn{3}{|c|}{ Anger } \\
\hline 14 & $\begin{array}{l}\text { The Legend of Bhagat Singh (English man saying } \\
\text { bloody Indian scene) }\end{array}$ & 0.48 \\
\hline 12 & Shaurya (Kay Kay Menon Interrogation) & 0.39 \\
\hline
\end{tabular}

Table 4: Emotion clusters with probability: In this table we present two stimuli for each emotion cluster with the probability quantifying the preference by the participants in choosing the emotional cluster for the stimulus.. Ratings provided by our participants are comparable with provided by Gross and Levenson (1995). For the list of all stimuli along with the probability of eliciting an emotion cluster see Table S8.

\section{Glossary}

\begin{tabular}{c|c} 
Full Form & Acronym \\
\hline International Affective Picture System & IAPS \\
Western, Educated, Industrialized, Rich and Democratic & WEIRD \\
principal Component Analysis & PCA \\
High Valence High Arousal & HVHA \\
Low Valence High Arousal & LVHA \\
Low Valence Low Arousal & LVLA \\
High Valence Low Arousal & HVLA \\
\hline
\end{tabular}

Table 5: Glossary 


\section{Acknowledgments}

We thank Vaughn Becker from Arizona State University for his suggestions in improving the manuscript. We also thank Mr Amit Tiwari for helping us during the study to monitor the experiment and Mr Pravin Srivastav and Ms Anandpreet Kaur for helping us get a significant number of participants.

\section{Author Contribution}

SM: Conceptualization, Data curation, Formal analysis, Investigation, Methodology, Project administration, Resources, Software, Visualization, Writing-original draft; NS: Project administration, Supervision, Validation, Methodology, Writing-review \& editing; MA: Investigation; UST: Project administration, Resources, Supervision, Funding acquisition.

\section{Declarations}

\section{Funding}

The authors have no relevant financial or non-financial interests to disclose.

\section{Conflicts of interest/Competing interests}

The authors have no competing interests to declare that are relevant to the content of this article.

\section{Ethics approval}

Ethical approval is taken from the Institutional Review Board, University of Allahabad.

\section{Consent to participate}

Informed consent was obtained from all individual participants included in the study.

\section{Availability of data and materials}

The dataset generated during the current study and the code used for the analysis are available in the Open Science Framework repository (https:// osf.io/m8dwz/?view_only=d07610b2a87146c9956b754228f6baf4).

\section{References}

Baveye, Y., Dellandrea, E., Chamaret, C., Chen, L. (2015). Liris-accede: A video database for affective content analysis. IEEE Transactions on Affective Computing, 6(1), 43-55. 
10.1109/TAFFC.2015.2396531

Butler, E.A., Lee, T.L., Gross, J.J. (2007). Emotion regulation and culture: Are the social consequences of emotion suppression culture-specific? Emotion, 7(1), 30.

Carvalho, S., Leite, J., Galdo-Álvarez, S., Gonçalves, O.F. (2012). The emotional movie database (emdb): A self-report and psychophysiological study. Applied psychophysiology and biofeedback, 37(4), 279-294.

$10.1007 /$ s10484-012-9201-6

Dhaka, S., \& Kashyap, N. (2017). Explicit emotion regulation: Comparing emotion inducing stimuli. Psychological Thought, 10(2), 303-314.

$10.5964 /$ psyct.v10i2.240

Gabert-Quillen, C.A., Bartolini, E.E., Abravanel, B.T., Sanislow, C.A. (2015). Ratings for emotion film clips. Behavior research methods, 47(3), 773787 .

10.3758/s13428-014-0500-0

Gilman, T.L., Shaheen, R., Nylocks, K.M., Halachoff, D., Chapman, J., Flynn, J.J., ... Coifman, K.G. (2017). A film set for the elicitation of emotion in research: A comprehensive catalog derived from four decades of investigation. Behavior research methods, 49(6), 2061-2082.

10.3758/s13428-016-0842-x

Gross, J.J., \& Levenson, R.W. (1995). Emotion elicitation using films. Cognition E emotion, 9(1), 87-108.

$10.1080 / 02699939508408966$

Henrich, J., Heine, S.J., Norenzayan, A. (2010). The weirdest people in the world? Behavioral and brain sciences, 33(2-3), 61-83.

Hewig, J., Hagemann, D., Seifert, J., Gollwitzer, M., Naumann, E., Bartussek, D. (2005). A revised film set for the induction of basic emotions. Cognition and emotion, 19(7), 1095.

$10.1080 / 02699930541000084$ 
Hogan, P.C. (2009). Understanding indian movies: culture, cognition, and cinematic imagination. University of Texas Press.

Jenkins, L.M., \& Andrewes, D.G. (2012). A new set of standardised verbal and non-verbal contemporary film stimuli for the elicitation of emotions. Brain Impairment, 13(2), 212-227.

\subsection{7/BrImp.2012.18}

Koelstra, S., Muhl, C., Soleymani, M., Lee, J.-S., Yazdani, A., Ebrahimi, T., ... Patras, I. (2011). Deap: A database for emotion analysis; using physiological signals. IEEE transactions on affective computing, 3(1), 18-31.

Lim, N. (2016). Cultural differences in emotion: differences in emotional arousal level between the east and the west. Integrative medicine research, 5(2), 105-109.

\subsection{6/j.imr.2016.03.004}

Lohani, M., Gupta, R., Srinivasan, N. (2013). Cross-cultural evaluation of the international affective picture system on an indian sample. Psychological Studies, 58(3), 233-241.

Mishra, S., Srinivasan, N., Tiwary, U.S. (2022). An affective video database using multimedia content analysis rated on indian samples. arXiv. Retrieved from https://arxiv.org/abs/2210.09785 10.48550/ARXIV .2210 .09785

Molho, C., Tybur, J.M., Güler, E., Balliet, D., Hofmann, W. (2017). Disgust and anger relate to different aggressive responses to moral violations. Psychological science, 28(5), 609-619.

Paltoglou, G., \& Thelwall, M. (2012). Seeing stars of valence and arousal in blog posts. IEEE Transactions on Affective Computing, 4(1), 116-123.

\subsection{9/T-AFFC.2012.36}

Philippot, P. (1993). Inducing and assessing differentiated emotion-feeling states in the laboratory. Cognition and emotion, 7(2), 171-193.

Ray, R., \& Gross, J. (2007). Emotion elicitation using films in handbook of emotion elicitation and assessment (eds coan, a. E allen, jj b.) 9-28. 
Oxford University Press.

Russell, J.A. (1980). A circumplex model of affect. Journal of personality and social psychology, 39(6), 1161.

Samson, A.C., Kreibig, S.D., Soderstrom, B., Wade, A.A., Gross, J.J. (2016). Eliciting positive, negative and mixed emotional states: A film library for affective scientists. Cognition and emotion, 30(5), 827-856.

$10.1080 / 02699931.2015 .1031089$

Schaefer, A., Nils, F., Sanchez, X., Philippot, P. (2010). Assessing the effectiveness of a large database of emotion-eliciting films: A new tool for emotion researchers. Cognition and emotion, 24(7), 1153-1172.

$10.1080 / 02699930903274322$

Shott, S. (1979). Emotion and social life: A symbolic interactionist analysis. American journal of Sociology, 84(6), 1317-1334.

Srinivasan, N., \& Gupta, R. (2010). Emotion-attention interactions in recognition memory for distractor faces. Emotion, 10(2), 207.

Srinivasan, N., \& Kumar, D. (2012). The vividness of happiness in dynamic facial displays of emotion.

Turner, J.H., \& Stets, J.E. (2005). The sociology of emotions. Cambridge University Press.

Von Leupoldt, A., Rohde, J., Beregova, A., Thordsen-Sörensen, I., Zur Nieden, J., Dahme, B. (2007). Films for eliciting emotional states in children. Behavior Research Methods, 39(3), 606-609.

10.3758/bf03193032

Warriner, A.B., Kuperman, V., Brysbaert, M. (2013). Norms of valence, arousal, and dominance for 13,915 english lemmas. Behavior research methods, 45(4), 1191-1207.

Zupan, B., \& Eskritt, M. (2020). Eliciting emotion ratings for a set of film clips: A preliminary archive for research in emotion. The Journal of 
$22 \quad A F D I$

Social Psychology, 160(6), 768-789. 